\title{
Las habilidades de comunicación como factor preventivo del síndrome de Burnout en los profesionales de la salud
}

\author{
Communication skills: a preventive factor \\ in Burnout syndrome in health professionals
}

\author{
C. Leal-Costa ${ }^{1}$, J.L. Díaz-Agea ${ }^{1}$, S. Tirado-González ${ }^{2}$, J. Rodríguez-Marín², \\ C.J. van-der Hofstadt ${ }^{3}$
}

\section{RESUMEN}

Fundamento. Los profesionales de la salud son un colectivo que sufre altos niveles de estrés laboral. El objetivo de este estudio es analizar la relación entre habilidades de comunicación y síndrome de Burnout en profesionales sanitarios. La propuesta es demostrar empíricamente la hipótesis de que el hecho de que el profesional de la salud cuente con habilidades de comunicación contribuye a prevenir el síndrome de Burnout.

Material y método. Se plantea un estudio observacional, analítico, transversal en una muestra de 927 profesionales sanitarios, 197 médicos (21,3\%), 450 enfermeras $(48,5 \%)$ y 280 auxiliares de enfermería (30,2\%). Se han usado la Escala sobre Habilidades de Comunicación en Profesionales de la Salud (EHC-PS) y el Maslach Burnout Inventory Human Services Survey (MBI-HSS).

Resultados. Se obtiene una correlación negativa y estadísticamente significativa entre las diferentes dimensiones de las habilidades de comunicación y las dimensiones agotamiento emocional y despersonalización del burnout. Por otro lado, se observa una correlación positiva y estadísticamente significativa entre las dimensiones de las habilidades de comunicación y la dimensión realización personal en el trabajo.

Conclusiones. Se ha encontrado evidencia de que las habilidades de comunicación de los profesionales de la salud protegen y amortiguan el síndrome de Burnout.

Palabras clave. Burnout. Comunicación. Profesionales de la salud. Hospital. Atención Primaria.

\begin{abstract}
Background. Health professionals are a group that suffers high levels of job stress and burnout. The aim of this study is to demonstrate empirically that the healthcare count on communication skills helps prevent Burnout Syndrome.
\end{abstract}

Method. An observational, analytical, cross-sectional study was proposed, involving a sample of 927 health professionals (197 doctors, 450 nurses and 280 auxiliary nurses). Participants completed questionnaires measuring communication skills in health care (EHC-PS) and the Maslach Burnout Inventory Human Services Survey (MBI-HSS).

Results. A negative and statistically significant correlation between the different dimensions of communication skills and emotional exhaustion and depersonalization dimensions of burnout was obtained. On the other hand, a positive and statistically significant correlation between the dimensions of communication skills and the personal accomplishment dimension of burnout was observed.

Conclusions. It was shown that the communication skills of health professionals provide protection from and cushion Burnout Syndrome.

Keywords. Burnout. Communication. Health Professionals. Hospital. Primary Health Care.
1. Facultad de Enfermería. Universidad Católica de Murcia.

2. Departamento de Psicología de la Salud. Universidad Miguel Hernández.

3. Unidad de Psicología Clínica de la Salud. Hospital General Universitario de Alicante.

\section{Correspondencia:}

César Leal Costa

Universidad Católica de Murcia

Campus de los Jerónimos s/n

30107. Guadalupe (Murcia)

E-mail: cleal@ucam.edu

Recepción: 7 de noviembre de 2014

Aceptación provisional: 5 de marzo de 2015

Aceptación definitiva: 8 de abril de 2015 


\section{INTRODUCCIÓN}

Los profesionales de la salud son uno de los colectivos que sufre altos niveles de estrés laboral, lo que genera un problema que afecta tanto al ámbito individual como a otros niveles (profesional, institucional y social) ${ }^{1}$.

El burnout es un síndrome que fue descrito por primera vez en 1974 por Herbert Freudenberger, y utilizado por primera vez públicamente por Maslash en 1976. En español se le puede denominar "síndrome del quemado en el trabajo", y se define como un estado de agotamiento y desgaste laboral, que, según se desprende de la literatura, afecta principalmente a los profesionales de servicios y asistencia a los demás, sobre todo profesionales de la enseñanza y sanitarias. Los componentes principales del burnout son el agotamiento emocional, la despersonalización y la baja realización personal o logro. En el ámbito laboral, el burnout genera insatisfacción laboral, deterioro del ambiente laboral, disminución de la calidad del trabajo, absentismo, abandono de la profesión y adopción de posturas pasivo-agresivas con los pacientes ${ }^{1-5}$.

El personal sanitario se enfrenta constantemente a una tarea compleja en la que influyen diversos estresores específicos de la profesión que suponen una alta implicación emocional y los relacionados con la organización del trabajo, como: exceso de estimulación aversiva (enfrentarse frecuentemente a situaciones de sufrimiento, muerte, dolor y pérdida), contacto continuo con enfermos (que exige un cierto grado de implicación para establecer una relación), la frustración de no poder curar, objetivo para el que han sido entrenados, etc. . $^{1,6,7}$.

Las estrategias para la intervención encaminadas a evitar el Burnout, deben contemplar tres niveles: 1) el nivel individual hace referencia a cómo los trabajadores deben desarrollar estrategias personales de índole cognitivo-conductual, y con carácter autoevaluativo, para eliminar o reducir fuentes de estrés, 2) el grupal incluye potenciar la formación en habilidades de comunicación y apoyo social de los profesionales, 3) el organizacional propone eliminar o disminuir los estresores del entorno laboral ${ }^{1,8}$.
Así, el hecho de que el profesional de la salud cuente con unas adecuadas habilidades de comunicación, puede contribuir a que se sienta más seguro, más competente $\mathrm{y}$ fomente las relaciones interpersonales con los pacientes y sus propios colegas y, así, prevenga, amortigüe y reduzca sus experiencias de estrés laboral crónico ${ }^{9-11}$.

En ausencia de estudios que demuestren cómo influye el que los profesionales de la salud tengan unas adecuadas habilidades de comunicación, y cómo se relacionan éstas con el síndrome de Burnout, no podemos establecer relaciones respaldadas empíricamente. Sin embargo, sí podemos establecer algunas hipótesis sobre algunas de las relaciones que se pueden esperar teóricamente.

En este estudio se plantea que unas adecuadas habilidades de comunicación en los profesionales de la salud se relacionan con un menor agotamiento emocional y despersonalización, y una mayor realización personal en el trabajo.

El objetivo de este estudio es analizar la relación entre habilidades de comunicación y síndrome de Burnout en profesionales sanitarios.

\section{MATERIAL Y MÉTODO}

Se plantea un estudio observacional, analítico, transversal, en una muestra de profesionales sanitarios (médicos, enfermeras y auxiliares de enfermería) del ámbito de la atención primaria y especializada.

Se realizó un muestreo por conveniencia, seleccionando dos hospitales generales, uno comarcal y uno privado de la Región de Murcia (HGU Virgen de la Arrixaca, HGU Reina Sofía, hospital Los Arcos y el hospital de Molina), y uno general, uno comarcal, y uno de gestión privada de la provincia de Alicante (HGU de Alicante, hospital Vega Baja y hospital de Torrevieja). Los centros de atención primaria en la región de Murcia se seleccionaron del área del HGU Virgen de la Arrixaca, y en la provincia de Alicante, del hospital Vega Baja.

De este modo, la muestra se clasificó en dos estratos, atención especializada y atención primaria. 
Como criterios de inclusión, todos los participantes debían 1) ser mayores de edad, 2) desempeñar su labor asistencial en el ámbito de atención especializada o atención primaria, 3) ser médico, enfermera o auxiliar de enfermería, y 4) firmar el consentimiento informado.

A todos ellos se les administró un cuestionario de variables sociodemográficas (edad, sexo, estado civil y número de hijos) y profesionales (categoría profesional, centro de trabajo, unidad de trabajo y meses trabajados en la unidad) diseñado ad hoc para el desarrollo del estudio; Escala sobre Habilidades de Comunicación en Profesionales de la Salud (EHC-PS) ${ }^{12,13}$. Escala autoadministrada compuesta por 18 ítems, con una escala de respuesta tipo Likert con 6 grados de respuesta. Esta escala ha demostrado tener unas adecuadas propiedades psicométricas en cuanto a análisis de ítems, estructura interna a través de análisis factorial confirmatorio, análisis de fiabilidad (consistencia interna mediante alfa de Cronbach en todas sus dimensiones y estabilidad temporal test-retest) y obtención de evidencias externas de validez. Las cuatro dimensiones que componen la escala son: Comunicación Informativa: compuesta por 6 ítems (5, 8, 9, 14, 17 y 18), refleja la manera que los profesionales de la salud tienen de obtener y proporcionar información en la relación clínica que establecen con los pacientes. Empatía: compuesta por 5 ítems $(2,4,6,11$ y 12$)$, refleja la capacidad de los profesionales de la salud de comprender los sentimientos de los pacientes y hacerlo evidente en la relación, así como la dimensión conductual de ésta, la actitud empática, compuesta por la escucha activa y la respuesta empática. Respeto: compuesta por 3 ítems (1, 3 y 15), evalúa el respeto que muestran los profesionales de la salud en la relación clínica que establecen con los pacientes. Habilidad social: los 4 ítems de esta dimensión $(7,10,13$ y 16) reflejan la habilidad que los profesionales de la salud tienen de ser asertivos o tener conductas socialmente habilidosas en la relación clínica que establecen con los pacientes.

El cuestionario Maslach Burnout Inventory Human Services Survey (MBI-HSS) ${ }^{14}$, en su versión adaptada a la población española es el más utilizado para evaluar la frecuencia e intensidad del síndrome de Burnout dentro del contexto laboral sanitario. Consta de 22 ítems, y en él se pueden diferenciar tres subescalas que miden, a su vez, las tres dimensiones que conforman el síndrome: agotamiento emocional (AE), despersonalización (DP) y realización personal en el trabajo (RP).

Para decidir en qué unidades se recogerían los datos, se realizó una clasificación de las unidades de la siguiente forma: Urgencias, UCI, planta hospitalización quirúrgica, planta hospitalización medicina interna, reanimación, quirófano, psiquiatría, planta hospitalización medicina interna (infecciosos, VIH), oncología, ginecologíaobstetricia, pediatría, UCI neonatal, oncología infantil, consultas externas y centros de atención primaria; se buscaba tener una muestra de todas las unidades mencionadas para tener representación de prácticamente todas las unidades hospitalarias y centros de atención primaria.

La muestra se recogió con la ayuda de las diferentes direcciones de los centros asistenciales que delegaron la tarea en los supervisores/coordinadores de enfermería. Se solicitó la participación voluntaria de los profesionales de la salud, ofreciéndoles las explicaciones oportunas para cumplimentar adecuadamente los instrumentos aplicados. A los participantes se les asignó una clave numérica para asegurar la confidencialidad de sus datos, cumplimentando los instrumentos en formato papel.

Se obtuvo la aprobación de los comités de ética de los centros para realizar el proyecto.

Para procesar la información se elaboró una base de datos con el programa informático SPSS@ v21, realizándose un análisis con diferentes tipos de pruebas estadísticas:

Estadística descriptiva (media, varianza, desviación típica) de las diferentes puntuaciones obtenidas por los profesionales de la salud, así como frecuencias y porcentajes de las variables sociodemográficas y profesionales.

Correlaciones bivariadas de Pearson entre las variables estudiadas, realizando 
los análisis según las variables categoría profesional y ámbito de trabajo.

Diferencias de medias (t-student $y$ ANOVA) entre las diferentes dimensiones del MBI y la EHC-PS y la variable sociodemográfica sexo y las variables profesionales categoría profesional y ámbito de trabajo.

Regresión lineal múltiple para conocer las variables predictoras de las tres dimensiones del burnout (agotamiento emocional, despersonalización y realización personal).

\section{RESULTADOS}

La muestra final estuvo compuesta por 927 profesionales de la salud, de los cuales, 197 eran médicos (21,3\%), 450 eran enfermeras $(48,5 \%)$ y 280 eran auxiliares de enfermería $(30,2 \%)$.

La media de edad de la muestra fue de 40,95 años con una desviación típica de 10,069 , siendo el $70,3 \%$ mujeres el $29,7 \%$ hombres. En la tabla 1 se muestran las frecuencias y porcentajes de las todas las variables sociodemográficas y profesionales de la muestra.

Tabla 1. Frecuencia y porcentaje de las variables sociodemográficas y profesionales de la muestra

\begin{tabular}{|c|c|}
\hline Variables & n (\%) \\
\hline \multicolumn{2}{|l|}{ Sexo: } \\
\hline Hombre & $275(29,7)$ \\
\hline Mujer & $652(70,3)$ \\
\hline \multicolumn{2}{|l|}{ Estado civil: } \\
\hline Soltero/a & $300(32,4)$ \\
\hline Casado/a & $554(59,8)$ \\
\hline Divorciado/a & $66(7,1)$ \\
\hline Viudo/a & $7(0,8)$ \\
\hline \multicolumn{2}{|l|}{ Edad (años): } \\
\hline$<20$ & $1(0,1)$ \\
\hline $20-30$ & $165(17,8)$ \\
\hline $31-40$ & $314(33,9)$ \\
\hline $41-50$ & $249(26,9)$ \\
\hline $51-60$ & $189(20,4)$ \\
\hline $61-70$ & $9(1)$ \\
\hline$>70$ & 0 \\
\hline \multicolumn{2}{|l|}{ Número de hijos: } \\
\hline 0 & $349(37,6)$ \\
\hline 1 & $168(18,1)$ \\
\hline 2 & $298(32,1)$ \\
\hline$>2$ & $112(12,1)$ \\
\hline \multicolumn{2}{|l|}{ Categoría profesional: } \\
\hline Médico & $197(21,3)$ \\
\hline Enfermera & $450(48,5)$ \\
\hline Auxiliar de enfermería & $280(30,2)$ \\
\hline \multicolumn{2}{|l|}{ Tiempo trabajado (meses): } \\
\hline$<60$ meses (5 años) & $231(24,9)$ \\
\hline $61-120$ meses (5-10 años) & $213(23)$ \\
\hline $121-180$ meses (10-15 años) & $145(15,9)$ \\
\hline $181-240$ meses (15-20 años) & $101(10,9)$ \\
\hline 241-300 meses (20-25 años) & $101(10,9)$ \\
\hline$>300$ meses $(>25$ años $)$ & $136(14,7)$ \\
\hline \multicolumn{2}{|l|}{ Ámbito de trabajo: } \\
\hline Atención especializada & $843(90,9)$ \\
\hline Atención primera & $84(9,1 \%)$ \\
\hline
\end{tabular}


El 90,9\% (843) de los respondedores trabajan en el ámbito de atención especializada, y 9,1\% (84) en el ámbito de atención primaria, obteniendo una representación de todos los estratos que se habían propuesto, aunque menor en atención primaria.

Se encontraron unas puntuaciones medias-altas en todas las dimensiones de la EHC-PS y una puntuación alta en la dimensión realización personal en el trabajo del MBI-HSS. Por otro lado, las puntuaciones en la dimensión agotamiento emocional son medias-bajas y bajas en la dimensión despersonalización del MBI-HSS (Tabla 2).
Para hacer una comparación sobre las diferentes dimensiones de las habilidades de comunicación y las dimensiones del "burnout" que poseen los sujetos de la muestra, en la figura 1 se reflejan las puntuaciones según la variable categoría profesional. Los médicos y las enfermeras puntúan más alto en las diferentes dimensiones de la EHC-PS que las auxiliares de enfermería, teniendo además menos niveles de agotamiento emocional (AE) y despersonalización (DP), y puntuando a la vez más alto en realización personal en el trabajo (RP).

Tabla 2. Estadísticos descriptivos (media y desviación típica) de las puntuaciones de la muestra en cada una de las dimensiones de la EHC-PS y del MBI-HSS del total de la muestra y según la variable categoría profesional

\begin{tabular}{lcccccccc} 
& \multicolumn{2}{c}{$\begin{array}{c}\text { Médicos } \\
(\mathbf{n = 1 9 7})\end{array}$} & $\begin{array}{c}\text { Enfermeras } \\
(\mathbf{n = 4 5 0 )}\end{array}$ & \multicolumn{2}{c}{$\begin{array}{c}\text { Auxiliar de } \\
\text { enfermería } \\
(\mathbf{n = 2 8 0})\end{array}$} & $\begin{array}{c}\text { Total } \\
(\mathbf{n = 9 2 7})\end{array}$ \\
\hline M & DT & M & DT & M & DT & M & DT \\
\hline Comunicación Informativa & 23,56 & 3,81 & 23,60 & 3,70 & 21,80 & 4,47 & 23,05 & 4,05 \\
\hline Respeto & 30,92 & 3,64 & 30,78 & 3,76 & 28,25 & 4,71 & 30,04 & 4,21 \\
\hline Habilidad social & 15,23 & 2,06 & 15,27 & 2,15 & 14,11 & 2,61 & 14,91 & 2,34 \\
\hline Agotamiento emocional & 17,78 & 3,34 & 17,05 & 3,47 & 14,61 & 4,16 & 16,47 & 3,87 \\
\hline Despersonalización & 19,17 & 11,04 & 18,60 & 10,60 & 19,64 & 12,02 & 19,03 & 11,14 \\
\hline Realización personal en el trabajo & 40,05 & 7,87 & 40,88 & 7,30 & 39,53 & 8,92 & 40,29 & 7,95 \\
\hline
\end{tabular}

M: Media; DT: Desviación típica.
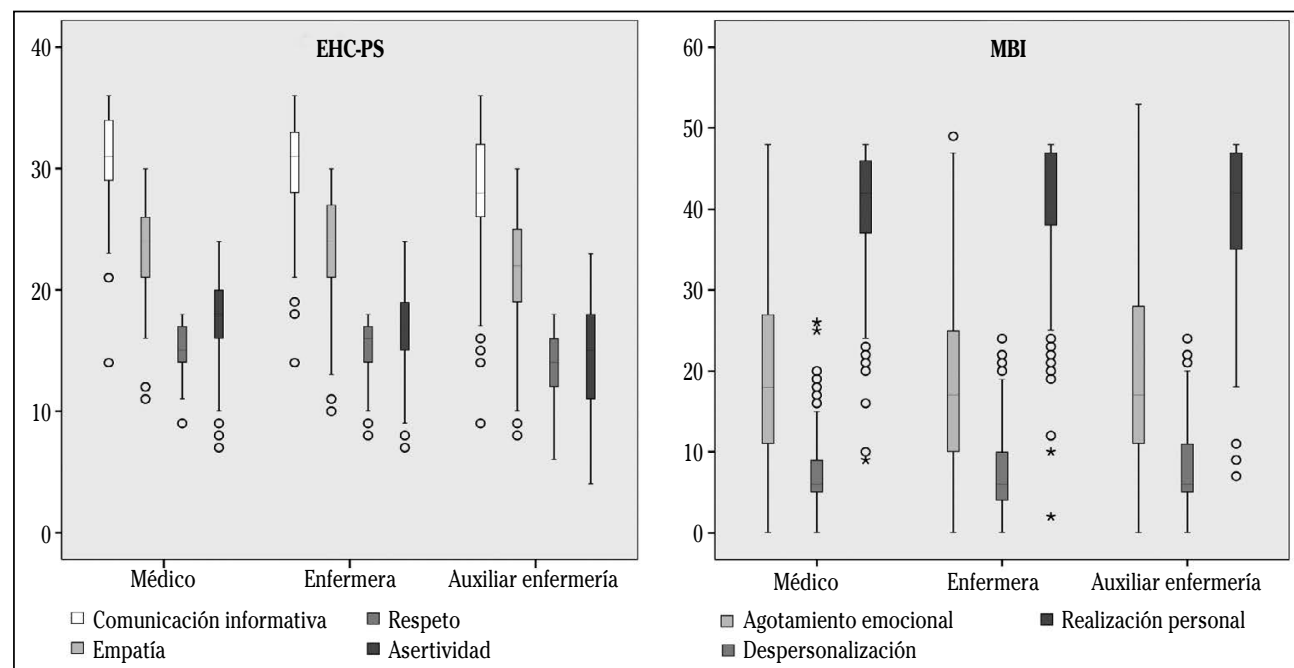

Figura 1. Puntuación de los profesionales de la salud en la EHC-PS y el MBI. Los diagramas de caja indican la mediana, el rango intercuartílico, valores máximos y mínimos y valores que son atípicos o extremos. 
Tabla 3. Correlaciones bivariadas de Pearson entre las variables sociodemográficas (edad, sexo, $n^{\circ}$ de hijos y tiempo trabajado), las dimensiones de las habilidades de comunicación (Comunicación informativa, empatía, respeto y habilidad social) y las dimensiones del burnout (agotamiento emocional, despersonalización y realización personal) según la variable categoría profesional y el ámbito del profesional

\begin{tabular}{|c|c|c|c|c|c|c|c|c|c|c|c|}
\hline & Edad & Sexo & $\mathrm{n}^{0}$ hijos & T Trab & Com inf & Emp & Resp & H Soc & $\mathrm{AE}$ & DP & $\mathbf{R P}$ \\
\hline \multicolumn{12}{|l|}{ Médicos } \\
\hline Edad & - & & & & & & & & & & \\
\hline Sexo & $0,275^{\text {* * }}$ & - & & & & & & & & & \\
\hline $\mathrm{n}^{0}$ hijos & $0,621^{\text {** }}$ & $0,165^{*}$ & - & & & & & & & & \\
\hline T Trab & $0,880^{* *}$ & $0,259^{* *}$ & $0,517^{\star \star}$ & - & & & & & & & \\
\hline Com inf & 0,085 & 0,095 & 0,043 & 0,096 & - & & & & & & \\
\hline Emp & 0,027 & 0,127 & 0,040 & 0,069 & $0,671^{* *}$ & - & & & & & \\
\hline Resp & 0,137 & 0,098 & 0,073 & $0,151^{*}$ & $0,667^{\text {** }}$ & $0,567^{* *}$ & - & & & & \\
\hline H Soc & 0,092 & 0,123 & 0,004 & $0,148^{*}$ & $0,448^{* *}$ & $0,433^{* *}$ & $0,389^{\text {* * }}$ & - & & & \\
\hline $\mathrm{AE}$ & 0,022 & 0,090 & 0,037 & 0,049 & $0,324^{* *}$ & $0,290^{* *}$ & $0,322^{\text {* * }}$ & $0,317^{* *}$ & - & & \\
\hline DP & 0,121 & 0,169 & 0,125 & 0,138 & $0,433^{* *}$ & $0,440^{* *}$ & $0,411^{\text {* * }}$ & $0,309^{* *}$ & $0,322^{* *}$ & - & \\
\hline RP & 0,120 & 0,086 & 0,089 & 0,085 & $0,510^{* *}$ & $0,556^{* *}$ & $0,473^{* *}$ & $0,327^{\text {* * }}$ & $0,435^{* *}$ & $0,561^{\text {* * }}$ & - \\
\hline
\end{tabular}

\section{Enfermeras}

\begin{tabular}{|c|c|c|c|c|c|c|c|c|c|c|}
\hline Edad & - & & & & & & & & & \\
\hline Sexo & $0,156^{* *}$ & - & & & & & & & & \\
\hline $\mathrm{n}^{\mathrm{o}}$ hijos & $0,576^{* *}$ & $0,135^{* *}$ & - & & & & & & & \\
\hline T Trab & $0,878^{* *}$ & $0,140^{* *}$ & $0,480^{\text {** }}$ & - & & & & & & \\
\hline Com_inf & 0,146 & 0,041 & 0,064 & $0,107^{*}$ & - & & & & & \\
\hline Emp & 0,074 & 0,047 & 0,048 & 0,070 & $0,718^{* *}$ & - & & & & \\
\hline Resp & 0,116 & 0,062 & $0,160^{* *}$ & 0,140 ** & $0,603^{* *}$ & $0,590^{* *}$ & - & & & \\
\hline H Soc & 0,041 & 0,037 & 0,052 & 0,051 & $0,437^{\text {* * }}$ & 0,397 ** & $0,366^{* *}$ & - & & \\
\hline $\mathrm{AE}$ & 0,109 & 0,003 & 0,076 & 0,052 & $0,382^{* *}$ & $0,318^{* *}$ & $0,371^{* *}$ & 0,341 ** & - & \\
\hline DP & 0,111 & 0,035 & 0,058 & $0,098^{*}$ & $0,381^{\text {* * }}$ & $0,306^{* *}$ & $0,375^{* *}$ & $0,273^{* *}$ & $0,287^{* *}$ & - \\
\hline RP & 0,068 & 0,026 & 0,091 & 0,023 & $0,517^{* *}$ & $0,511^{* *}$ & $0,409 * *$ & 0,332 * * & 0,379 ** & $0,526^{* *}$ \\
\hline
\end{tabular}

Auxiliar de Enfermería

\begin{tabular}{|c|c|c|c|c|c|c|c|c|c|c|c|}
\hline Edad & - & & & & & & & & & & \\
\hline Sexo & $0,237^{* *}$ & - & & & & & & & & & \\
\hline $\mathrm{n}^{0}$ hijos & $0,414^{* *}$ & 0,078 & - & & & & & & & & \\
\hline T Trab & $0,691^{* *}$ & $0,197^{* *}$ & $0,287^{* *}$ & - & & & & & & & \\
\hline Com_inf & 0,120 & 0,114 & 0,119 & 0,059 & - & & & & & & \\
\hline Emp & 0,120 & 0,042 & 0,052 & 0,084 & $0,701^{* *}$ & - & & & & & \\
\hline Resp & 0,131 & 0,088 & 0,112 & 0,085 & $0,614^{* *}$ & $0,583^{* *}$ & - & & & & \\
\hline H Soc & 0,051 & 0,123 & 0,034 & 0,060 & $0,425^{\star *}$ & $0,407^{* *}$ & $0,373^{* *}$ & 1 & & & \\
\hline $\mathrm{AE}$ & 0,035 & 0,025 & 0,006 & 0,068 & $0,389 * *$ & 0,381 * * & $0,314^{* *}$ & $0,229 * *$ & - & & \\
\hline DP & 0,036 & 0,010 & 0,044 & 0,029 & $0,348^{* *}$ & $0,325^{* *}$ & $0,314^{* *}$ & $0,263^{* *}$ & $0,428^{* *}$ & - & \\
\hline $\mathrm{RP}$ & 0,009 & 0,048 & 0,022 & 0,028 & $0,448^{* *}$ & 0,399 ** & $0,363^{\text {* * }}$ & $0,247^{\text {* * }}$ & $0,520^{\text {** }}$ & $0,584^{* *}$ & - \\
\hline
\end{tabular}




\begin{tabular}{|c|c|c|c|c|c|c|c|c|c|c|c|}
\hline & Edad & Sexo & $\mathrm{n}^{\mathbf{0}}$ hijos & T Trab & Com inf & Emp & Resp & H Soc & $\mathrm{AE}$ & DP & $\mathbf{R P}$ \\
\hline \multicolumn{12}{|c|}{ Centros de Atención Primaria } \\
\hline Edad & - & & & & & & & & & & \\
\hline Sexo & $0,362^{* *}$ & - & & & & & & & & & \\
\hline $\mathrm{n}^{0}$ hijos & $0,617^{\text {** }}$ & 0,211 & - & & & & & & & & \\
\hline T Trab & $0,838^{* *}$ & 0,212 & $0,517^{\text {* * }}$ & - & & & & & & & \\
\hline Com_inf & $0,172^{*}$ & 0,177 & 0,094 & $0,193^{*}$ & - & & & & & & \\
\hline Emp & $0,196^{*}$ & 0,145 & 0,143 & $0,276^{*}$ & $0,620^{* *}$ & - & & & & & \\
\hline Resp & $0,155^{*}$ & 0,146 & 0,142 & $0,273^{*}$ & $0,589 * *$ & $0,630^{* *}$ & - & & & & \\
\hline H Soc & $0,211^{*}$ & 0,049 & 0,123 & $0,309^{* *}$ & $0,316^{* *}$ & $0,361^{\text {** }}$ & $0,399^{* *}$ & - & & & \\
\hline $\mathrm{AE}$ & $0,162^{*}$ & $0,155^{*}$ & 0,107 & $0,159^{*}$ & 0,250 ** & $0,290^{* *}$ & $0,268^{* *}$ & $0,247^{* *}$ & - & & \\
\hline DP & $0,178^{*}$ & 0,030 & 0,020 & $0,208^{*}$ & $0,255^{* *}$ & $0,268^{* *}$ & $0,215^{* *}$ & 0,279 ** & $0,346^{* *}$ & - & \\
\hline $\mathrm{RP}$ & $0,188^{*}$ & $0,240^{*}$ & 0,007 & $0,190^{*}$ & $0,357^{* *}$ & $0,307^{* *}$ & $0,405^{* *}$ & $0,279^{* *}$ & $0,457^{* *}$ & $0,552^{* *}$ & - \\
\hline
\end{tabular}

\section{Hospitalización}

\begin{tabular}{|c|c|c|c|c|c|c|c|c|c|c|}
\hline Edad & - & & & & & & & & & \\
\hline Sexo & 0,013 & - & & & & & & & & \\
\hline $\mathrm{n}^{\circ}$ hijos & 0,531 ** & 0,021 & - & & & & & & & \\
\hline T Trab & $0,787^{* *}$ & 0,005 & $0,398^{* *}$ & - & & & & & & \\
\hline Com_inf & $0,204^{* *}$ & $0,086^{*}$ & $0,124^{* *}$ & $0,216^{* *}$ & - & & & & & \\
\hline Emp & $0,185^{\text {** }}$ & 0,003 & $0,079 *$ & $0,195^{\text {* * }}$ & $0,722^{* *}$ & - & & & & \\
\hline Resp & 0,209 * * & $0,105^{* *}$ & $0,150^{* *}$ & 0,231 * * & $0,644^{* *}$ & $0,600^{* *}$ & - & & & \\
\hline H Soc & $0,168^{* *}$ & $0,151^{* *}$ & $0,073^{*}$ & $0,178^{*}$ & $0,494^{* *}$ & $0,446^{* *}$ & $0,415^{\text {** }}$ & - & & \\
\hline $\mathrm{AE}$ & $0,145^{*}$ & 0,017 & $0,126^{*}$ & $0,126^{*}$ & $0,393^{* *}$ & 0,349 ** & $0,367^{* *}$ & $0,308^{* *}$ & - & \\
\hline DP & $0,193^{\text {** }}$ & 0,048 & $0,168^{*}$ & $0,146^{*}$ & $0,354^{* *}$ & $0,369^{* *}$ & $0,327^{\text {* * }}$ & $0,302^{\text {** }}$ & $0,348^{* *}$ & - \\
\hline $\mathrm{RP}$ & $0,182^{*}$ & 0,014 & $0,185^{*}$ & $0,164^{*}$ & 0,496 ** & $0,494^{* *}$ & $0,407^{* *}$ & $0,360^{* *}$ & $0,442^{* *}$ & 0,560 * * \\
\hline
\end{tabular}

T Trab: Tiempo trabajado; Com_inf: Comunicación informativa; Emp: Empatía; Resp: Respeto; H Soc: Habilidad social; AE: Agotamiento emocional; DP: Despersonalización; RP: Realización personal en el trabajo. ${ }^{*} \mathrm{p}<0,05 ;{ }^{*} \mathrm{p}<0,01$

En cuanto al análisis de las correlaciones bivariantes según la categoría profesional y el ámbito de trabajo (Tabla 3), se observa una correlación negativa y estadísticamente significativa entre las diferentes dimensiones de las habilidades de comunicación en los profesionales de la salud y las dimensiones AE y DP de "burnout". Por otro lado, se observa una correlación positiva y estadísticamente significativa entre las dimensiones de las habilidades de comunicación en los profesionales de la salud y la dimensión RP de "burnout". Además, se observa una correlación positiva y estadísticamente significativa entre las variables sociodemográficas edad, número de hijos y tiempo trabajado. Cabe destacar que en el análisis bivariante realizado en el ámbito de atención primaria se observa una correlación negativa y estadísticamente significativa entre las dimensiones de las habilidades de comunicación y la dimensión realización personal en el trabajo del burnout y las variables sociodemográficas edad y tiempo trabajado, y una correlación positiva y estadísticamente significativa entre las dimensiones del burnout agotamiento emocional y despersonalización y las variables sociodemográficas edad y tiempo trabajado.

El análisis de las diferencias de medias muestra que hay diferencias estadísticamente significativas entre: la dimensión empatía y la variable categoría profesional 
$\mathrm{F}(2,926)=19,918, \mathrm{p}=0,000$; la dimensión comunicación informativa y las variables sexo $\mathrm{t}_{925}=2,169, \mathrm{p}=0,030$ y categoría profesional $\mathrm{F}(2,926)=39,653, \mathrm{p}=0,000$; la dimensión respeto y las variables sexo $t_{925}=2,795, p=0,005$ y categoría profesional $\mathrm{F}(2,926)=24,988$, $\mathrm{p}=0,000$; la dimensión habilidad social y las variables sexo $t_{925}=4,391, p=0,000$ y categoría profesional $\mathrm{F}(2,926)=54,126, \mathrm{p}=0,000$. No se encontraron diferencias estadísticamente significativas entre la variable ámbito de trabajo y las dimensiones del MBI y la EHC-PS.

En los análisis de la regresión lineal múltiple se utilizaron las dimensiones del MBI (AE, DP, RP) como variables dependientes, y las variables sociodemográficas (edad, sexo y número de hijos), las variables profesionales (tiempo trabajado), y las dimensiones de la EHC-PS (comunicación informativa, empatía, respeto y habilidad social) como variables predictoras. Con relación al agotamiento emocional, las variables que mejor lo predijeron fueron la comunicación informativa, la empatía y el respeto $(\mathrm{R} 2=0,105 ; \mathrm{F}(3,923)=36,255 ; \mathrm{p}=0,000)$. Con respecto a la despersonalización $(\mathrm{R} 2=$ $0,144 ; \mathrm{F}(3,923)=51,862 ; \mathrm{p}=0,000)$ y a la rea- lización personal en el trabajo $(\mathrm{R} 2=0,276$; $\mathrm{F}(3,923)=117,310 ; \mathrm{p}=0,000)$, las variables que mejor los predijeron fueron las mismas (Tabla 4). Como se puede observar en la tabla 4, los coeficientes de regresión de las variables predictoras (comunicación informativa, empatía y respeto) son negativos cuando las variables dependientes son el agotamiento emocional y la despersonalización, y positivo, cuando la variable dependiente es la realización personal en el trabajo.

\section{DISCUSIÓN}

En este estudio se plantea analizar la relación entre habilidades de comunicación y síndrome de Burnout en una muestra heterogénea compuesta por los tres colectivos de profesionales de la salud más numerosos en nuestro sistema sanitario (auxiliares de enfermería, enfermeras y médicos).

La muestra ha obtenido una puntuación media-alta en todas dimensiones de las habilidades de comunicación, siendo los colectivos profesionales con puntuaciones más altas los médicos y las enfermeras. Comparando este estudio con otros de ca-

Tabla 4. Análisis de regresión lineal múltiple sobre las dimensiones del MBI (agotamiento emocional, despersonalización y realización personal en el trabajo)

\begin{tabular}{|c|c|c|c|c|c|}
\hline & $\mathbf{R}^{2}(\%)$ & $\mathbf{F}$ & B & $\mathbf{t}$ & $\mathbf{p}$ \\
\hline VD: Agotamiento emocional & 10,5 & 36,255 & & & 0,000 \\
\hline Constante & & & 44,822 & 17,056 & 0,000 \\
\hline Empatía & & & $-0,307$ & $-2,410$ & 0,016 \\
\hline Comunicación Informativa & & & $-0,452$ & $-3,550$ & 0,000 \\
\hline Respeto & & & $-0,412$ & $-2,055$ & 0,040 \\
\hline VD: Despersonalización & 14,4 & 51,862 & & & \\
\hline Constante & & & 20,375 & 18,527 & 0,000 \\
\hline Empatía & & & $-0,155$ & $-2,915$ & 0,004 \\
\hline Comunicación Informativa & & & $-0,203$ & $-3,820$ & 0,000 \\
\hline Respeto & & & $-0,247$ & $-2,945$ & 0,003 \\
\hline VD: Realización Personal & 27,6 & 117,310 & & & \\
\hline Constante & & & 10,246 & 6,070 & 0,000 \\
\hline Empatía & & & 0,462 & 5,648 & 0,000 \\
\hline Comunicación Informativa & & & 0,473 & 5,783 & 0,000 \\
\hline Respeto & & & 0,353 & 2,738 & 0,006 \\
\hline
\end{tabular}


racterísticas semejantes ${ }^{15,16}$ se observa que los resultados son similares, sin embargo, la herramienta usada en otros estudios ha sido la Escala de Habilidades Sociales (EHS) de Gismero ${ }^{17}$, que solo evalúa habilidad social, y está validada en población general, no siendo específica para profesionales sanitarios.

Otro estudio ${ }^{9}$ propone que el entrenamiento en habilidades de comunicación en médicos de atención primaria podría prevenir el síndrome de Burnout, concluyendo que las habilidades de comunicación estarían asociadas a un menor padecimiento del síndrome de Burnout. Sin embargo, en este estudio no se realizó ninguna evaluación de las habilidades de comunicación en los participantes de la muestra. Esto pone de manifiesto la dificultad metodológica que supone tener evidencias empíricas de cómo las habilidades de comunicación inciden de manera positiva o negativa en las dimensiones del Burnout.

En este sentido Epstein $^{10}$ afirma que unas adecuadas habilidades de comunicación por parte de los profesionales de la salud incidirían en un menor padecimiento del síndrome de Burnout y por tanto, en la prevención de éste sin llegar a demostrarlo empíricamente. Creemos que este trabajo contribuye a aportar evidencias empíricas de que las habilidades de comunicación en los profesionales de la salud son un factor preventivo de padecer Burnout en consonancia con lo que otros autores defendían de forma teórica ${ }^{1,10,11,18}$.

En otro contexto sanitario, aunque no se ha estudiado directamente la influencia de las habilidades de comunicación en los profesionales de la salud como factor preventivo de padecer Burnout, se ha estudiado a través de la inteligencia emocional, ya que una de las dimensiones básicas de ésta es la gestión de las relaciones (asertividad, empatía, respeto, etc) ${ }^{19,20}$. Así, diversos estudios han demostrado que la inteligencia emocional se asocia significativamente con menos niveles de Burnout y mayor satisfacción en el trabajo ${ }^{21,22}$. Un estudio publicado recientemente, relaciona la empatía con la calidad de vida y el burnout ${ }^{23}$. Los resultados arrojados en el análisis bivariante (Ta- bla 3) y el análisis de regresión múltiple, están en consonancia con estos estudios, donde la varianza explicada por las dimensiones relacionales es semejante. Además, estos resultados son congruentes con la hipótesis planteada, obteniendo una relación negativa (tanto en el análisis bivariante como de regresión) entre las habilidades de comunicación y las dimensiones agotamiento emocional y despersonalización, y una relación positiva entre las habilidades de comunicación y la realización personal en el trabajo.

En cuanto a las diferencias de medias entre las puntuaciones de la muestra en las dimensiones de la EHC-PS y las dimensiones del MBI-HSS y la variable sociodemográfica sexo y las profesionales categoría profesional y ámbito de trabajo, se observa que los resultados arrojados muestran que existen diferencias significativas con las dimensiones de la EHC-PS, sin embargo, no se encuentran apenas diferencias con las dimensiones del MBI.

Como limitación del presente estudio, se deberían haber tenido en cuenta otras variables predictoras expuestas en otros $\operatorname{trabajos}^{23,24}$ que mejorarían la varianza explicada de las dimensiones del burnout (trabajar en zona urbana y rural, consumo de psicofármacos, presión asistencial, tipo de contrato, satisfacción de los profesionales y de los pacientes, calidad de vida, autoeficacia, autoestima, estrés, etc). Así, en futuros trabajos se debería dar un paso más e incluir más variables predictoras que explicasen las dimensiones del burnout en poblaciones más homogéneas de profesionales de la salud, incidiendo en las particularidades de cada ámbito de trabajo y de cada perfil profesional. Otra limitación importante del trabajo es la escasa muestra que se obtuvo del estrato "atención primaria”, por tanto, el patrón de asociación encontrado entre las habilidades de comunicación y el burnout con el tiempo trabajado y la edad, debería ser estudiado en otra muestra mayor, con un mayor número de variables predictoras.

Con los resultados obtenidos surge una doble reflexión. Por un lado la necesidad de entrenar a los profesionales en la ad- 
quisición de habilidades de comunicación, aumentando el peso que la calidad de las relaciones interpersonales (interacción humana) y las características individuales tienen en la asistencia sanitaria ${ }^{25}$. De ese modo se contribuiría a mejorar la salud de los trabajadores en el ámbito clínico. Por otro no debemos olvidar que, como ponen de manifiesto algunos estudios ${ }^{5,26-28}$, unas adecuadas habilidades de comunicación del profesional sanitario tienen su impronta en la satisfacción, reducción del estrés, adherencia al tratamiento y el sufrimiento de los pacientes.

Podemos concluir por tanto, que las habilidades de comunicación de los profesionales de la salud se relacionan con un menor agotamiento emocional y despersonalización, y una mayor realización personal en el trabajo. Por tanto, podemos afirmar, que unas adecuadas habilidades de comunicación ayudan a proteger y amortiguar el síndrome de Burnout en los profesionales sanitarios.

\section{BIBLIOGRAFÍA}

1. GiL-Monte PR. El síndrome de quemarse por el trabajo (Burnout). Madrid: Pirámide, 2005.

2. Apples A. Estrés laboral, agotamiento y enfermedad. En: Buendía J, editor. Estrés laboral y salud. Madrid: Biblioteca Nueva; 1998. p. 119-128.

3. AtANCE JC. Aspectos epidemiológicos del síndrome de Burnout en personal sanitario. Rev Esp Salud Publica 1997; 71: 293-303.

4. Olmedo M, Santed MA, Jiménez R, Gómez MD. El síndrome de Burnout: variables laborales, personales y psicopatologías asociadas. Psiquis 2001; 3: 117-129.

5. Pérez-Ciordia I, Guillén-Grima F, Aguinaga-Ontoso I. Satisfacción laboral y factores de mejora en profesionales de atención primaria. An Sist Sanit Navar 2013; 36: 253-262.

6. Mingote JC, Moreno B, Gálvez M. Desgaste profesional y salud de los profesionales médicos: revisión y propuestas de prevención. Med Clin 2004; 123: 265-270.

7. Zapf D, Seifert C, Schmutte B, Mertini H, Holz M. Emotion work and job stressors and their effects on Burnout. Psychol Health 2001; 5: 527-545.

8. Fuertes C, Arillo A, Arroyo P, Gaminde I, Pascual $\mathrm{P}$, Elcuaz $\mathrm{C}_{\mathrm{H}}$. Una experiencia de grupo de reflexión para el manejo de entrevistas clínicas difíciles. An Sist Sanit Navar 2013; 36: 455-466.

9. Cebrià J, Palma C, Segura J, Gracia R, Pérez J. El entrenamiento en habilidades de comunicación podría ser un factor preventivo del síndrome de Burnout en médicos de familia. Rev Psiquiatría Fac Med Barna 2006; 33: 34-40.

10. Epstein RM. Comunicación, Burnout y resultados clínicos: más preguntas que respuestas. Aten Primaria 2001; 27: 511-513.

11. Shimizu T, Mizoue T, Kubota S, Mishima N, Nagata S. Relationship between burnout and communication skill training among japanese hospital nurses: a pilot study. J Occup Health 2003; 45: 185-190.

12. Leal C, Tirado S, Rodríguez-Marín J, van-Der HoFSTADT CJ. Creación de la Escala sobre Habilidades de Comunicación en profesionales de la salud, EHC-PS. An Psicol 2015 (en prensa).

13. Leal C, TiRado S, Rodríguez-Marín J, VAN-DER Hofstadt CJ. Psychometric properties of the Health Professionals Communication Skills Scale (HP-CSS). Int J Clin Health Psychol 2015 (en prensa).

14. GIL-Monte PR. Factorial validity of the Maslach Burnout Inventory (MBI-HSS) among Spanish Professionals. Rev Saude Publica 2005; 39: 1-8.

15. LeAl C, Luján I, Gascón J, Ferrer L, van-der HofsTADT CJ. Habilidades sociales en los profesionales de urgencias y cuidados críticos de un hospital público comarcal. Enferm Intensiva 2010; 21: 136-141.

16. PADÉs A. Habilidades sociales en enfermería: propuesta de un programa de intervención. (Tesis doctoral). Universidad de las Islas Baleares. Mallorca; 2003.

17. Gismero E. EHS Escala de Habilidades Sociales. Manual. Madrid: TEA Publicaciones de Psicología Aplicada; 2010.

18. Bellón JA. Habilidades de Comunicación y utilización de las consultas de atención primaria. An Sist Sanit Navar 2001; 24 (Supl. 2): 7-14.

19. Giardini A, Frese M. Reducing the negative effects of emotion work in service occupations: Emotional competence as a psychological resource. J Occup Health Psychol 2006; 11: 63-75.

20. Matthews G, Zeidner M. Emotional Intelligence, Adaptation to Stressful. Encounters, and Health Outcomes. En: Bar-On R, Parker JDA, editors. The Handbook of Emotional Intelligence. Theory, development, assessment, and application at home, School, and in the workplace. San Francisco, Ca: Jossey-Bass; 2000. p. 459-489. 
21. Hui-Ching W, Chao-Ming H, Yi-Tien L, Yu-Yen C, Cheng-Yo Y, Chi-Chang C et al. Associations between emotional intelligence and doctor burnout, job satisfaction and patient satisfaction. Med Educ 2011; 45: 835-842.

22. Görgens-EkERMAns G, Brand T. Emotional Intelligence as a moderator in the stress-burnout relationship: a questionnaire study on nurses. J Clin Nurs 2012; 21: 2275-2285.

23. Paro HBM, Silveira PS, Perotta B, Gannam S, EnNS SC, GiaXa RR et al. Empathy among medical students: is there a relation with quality of life and burnout? Plos One 2014; 9: 1-10.

24. Navarro D, Ayechu A, Huarte I. Prevalencia del síndrome del Burnout y factores asociados a dicho síndrome en los profesionales sanitarios de atención primaria. SEMERGEN 2014, en prensa.
25. Zoppi K, Epstein RM. ¿Es la comunicación una habilidad? Las habilidades comunicativas para mantener una buena relación. An Sist Sanit Navar 2001; 24 (Supl. 2): 23-31.

26. Díaz JL, Leal C, Martin M, Echevarría P. La comunicación entre el personal sanitario y los afectados por una amputación traumática. La sábana por encima. Rev Comun Salud 2013; 3: 5-19.

27. Sliwa JA, Makoul G, Betts H. Rehabilitationspecific communication skills training: improving the physician patient relationship. Am J Phys Med Rehabil 2002; 81: 126-132.

28. Dierssen-Sotos T, Rodríguez-Cundín P, RoblesGarcía M, Brugos-Llamazares V, Gómez-Acebo I, LlorCA J. Factores que determinan la alta satisfacción del usuario con la asistencia hospitalaria. An Sist Sanit Navar 2009; 32: 317-325. 